\title{
Multiprofessionelle Behandlungsansätze in der Alterstraumatologie
}

\author{
Norbert Pfundtner und Frank Blömker
}

Wie erreichen wir den älteren Patienten in einem Umfeld sich verändernder Rahmenbedingungen? Wie finden wir die richtige Therapie für die Patienten, die nur eingeschränkt mitarbeiten können? Diese Fragen können bei alleiniger Betrachtung der körperlichen Defizite nur unbefriedigend beantwortet werden. Multimorbidität und altersphysiologische Veränderungen lassen eine besondere Vorgehensweise in Diagnostik und Therapie notwendig werden. Für die Traumatologie führen sie zu erheblichen Konsequenzen: Erlaubt die Osteosynthese eine Übungsstabilität? Ist Vollbelastung möglich? Werden Hilfsmittel adäquat angepasst? Kann ich mit dem Patienten einzelne Therapieschritte verabreden? Muss ich in anderer Form Präventionsstrategien (z. B. Sturzprophylaxe/Balancetraining) in die Behandlung einbauen?
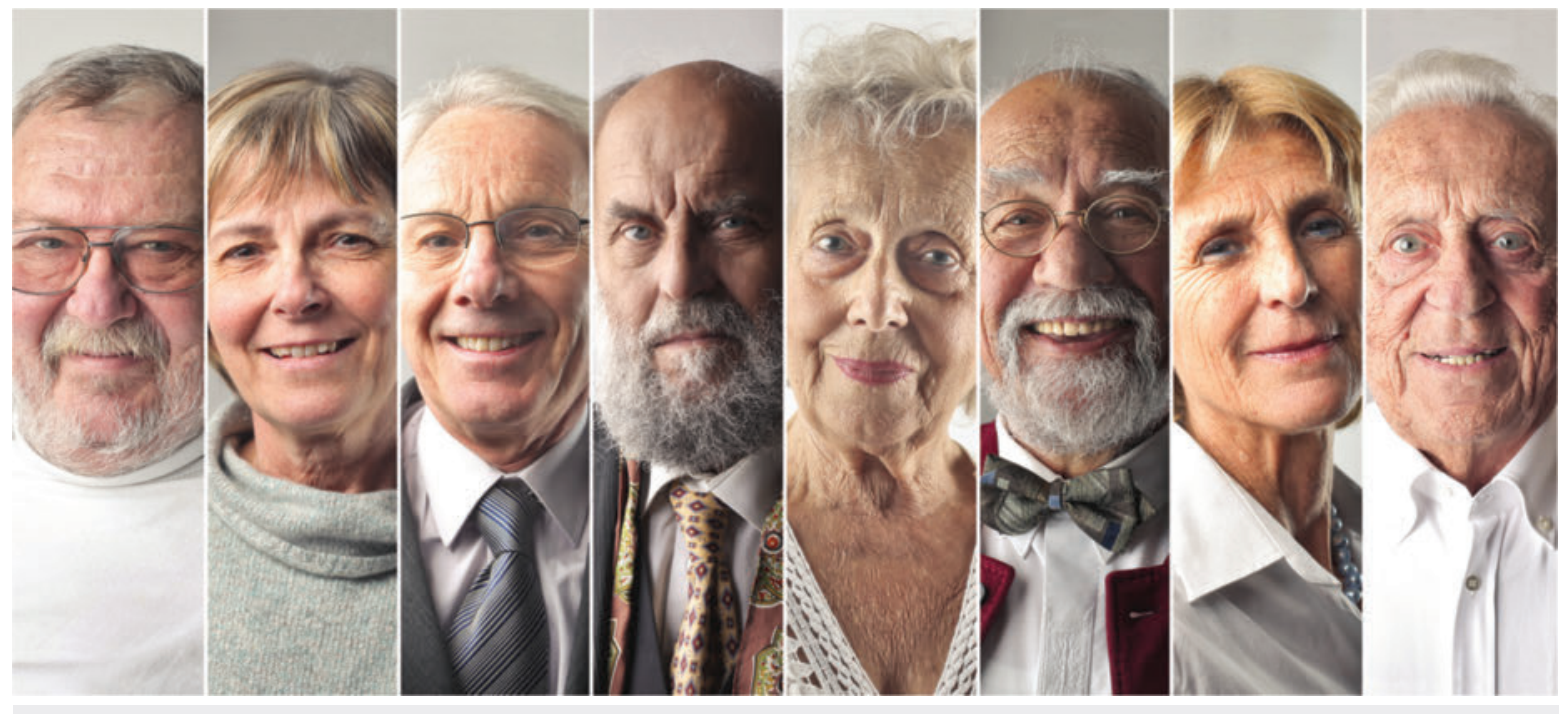

Autonomie, Wohlbefinden und das Erreichen persönlicher Lebensziele tragen zum optimalen Altern bei. (Quelle: Ollyy/ shutterstock.com; Symbolbild)

Alte und sehr alte Menschen nehmen in unterschiedlicher Ausprägung am gesellschaftlichen Leben teil und stellen andere Bedürfnisse und Schwerpunkte in ihrem Leben in den Vordergrund als junge Menschen. Für ein Altern mit weitreichender Autonomie, Wohlbefinden und dem Erreichen von persönlichen Lebenszielen sind die gesundheitliche Entwicklung und materielle Abhängigkeiten zu betrachten. Wichtig ist auch die soziale Verwurzelung im Sinne eines funktionierenden Netzwerks, das in der Lage ist, plötzliche oder schleichende Veränderungen der bisherigen Lebensplanung zu kompensieren. Das Alter ist also keine einheitlich geprägte Lebensphase und muss auch nicht mit Krankheiten assoziiert sein, jedoch steigt mit zunehmendem Alter die Wahrscheinlichkeit, Leistungseinbußen körperlicher oder geistiger Fähigkeiten, auch durch Krankheiten, zu erleben. 


\section{Demografische Alterung}

Seit den 1970er-Jahren ist die Lebenserwartung für 65-Jährige um etwa fünf Jahre gestiegen, für 80-Jährige um fast drei Jahre [35]. Im Jahr 2013 waren 20,8\% der Gesamtbevölkerung in Deutschland 65 Jahre oder älter, was etwa 17 Millionen Menschen entspricht. 4,4 Millionen Menschen waren über 80 Jahre alt (5,4\% der Gesamtbevölkerung), 2,1 Millionen 85 Jahre oder älter (2,6\% der Gesamtbevölkerung) [36].

Die Prognosen dazu sagen, dass bis Mitte der 2030er-Jahre die Gruppe der 65-Jährigen und bis Mitte der 2050er-Jahre die Gruppe der über 80-Jährigen weiter zunehmen werden [34]. Dies ist neben der hohen Lebenserwartung v.a. der Generation der Babyboomer und den anhaltend niedrigen Geburtenraten geschuldet [24]. Diese Veränderung der Altersstruktur zugunsten der höheren Altersgruppen wird demografische Alterung genannt.

Die Inanspruchnahme des Gesundheitswesens steigt mit der Zahl älterer Menschen. 96\% der Frauen und 97,7\% der Männer haben nach einer Befragung von 2013 in den vorangegangenen 12 Monaten einen Arzt aufgesucht [27]. Von den 19 Mio. Krankenhausfällen im Jahr 2013 entfielen 8,3 Mio. auf Menschen, die älter als 65 Jahre waren (4,5 Mio. Frauen, 3,8 Mio. Männer) [37], was gegenüber dem Jahr 2000 einem Anstieg von $20,6 \%$ bei den Frauen und $52 \%$ bei den Männern entspricht.

\section{Herausforderungen in Geriatrie und Alterstraumatologie}

\section{Multimorbidität}

Das steigende individuelle Krankheitsrisiko und die demografische Alterung führen immer häufiger dazu, dass altersbedingte chronische Erkrankungen gleichzeitig auftreten [14]. Wenn diese chronischen Erkrankungen sich akut verschlechtern oder auf eine neu hinzugekommene Akuterkrankung treffen, werden die medizinische Diagnostik und Therapie zunehmend komplex.

\section{DEFINITION}

Nach der aktuellen DEGAM-Leitlinie wird Multimorbidität durch das gleichzeitige Vorliegen von mindestens drei chronischen Erkrankungen definiert. Dabei muss keine der Erkrankungen zentrale Bedeutung haben. Über gemeinsame Risikofaktoren oder bei Folgeerkrankungen können Zusammenhänge zwischen den Krankheiten bestehen; das muss aber nicht sein. [33]
Die häufigsten Diagnosen bei den Frauen sind die Herzinsuffizienz, Krankheiten der Wirbelsäule und des Rückens sowie die koronare Herzkrankheit (KHK). Die Männer werden am häufigsten aufgrund einer KHK stationär behandelt, dann folgen Herzinsuffizienz und Herzrhythmusstörungen.

Geriatrische Patienten weisen dabei eine geriatrietypische Multimorbidität [4] sowie ein höheres Lebensalter (überwiegend 70 Jahre oder älter) auf, wobei die geriatrietypische Multimorbidität hierbei vorrangig vor dem kalendarischen Alter zu sehen ist. Sind die Patienten 80 Jahre und älter, ist typischerweise eine erhöhte Vulnerabilität gegeben, z.B. aufgrund des Auftretens von Komplikationen und Folgeerkrankungen, der Gefahr der Chronifizierung sowie des erhöhten Risikos eines Autonomieverlusts mit Verschlechterung des Selbsthilfestatus (Deutsche Gesellschaft für Geriatrie, www.dgg.de).

Eine geriatrietypische Multimorbidität ist gegeben, wenn mindestens zwei der folgenden Befunde vorliegen [4]:

- Immobilität

- Sturzneigung/Schwindel

- kognitive Defizite

- Inkontinenz

- Dekubitus

- Fehl-/Mangelernährung

- Flüssigkeits-/Elektrolythaushaltsstörung

- Depression/Angststörung

- Schmerz

- Sensibilitätsstörung

- herabgesetzte Belastbarkeit/Gebrechlichkeit

- Seh-/Hörstörung

- Medikationsproblem

- hohes Komplikationsrisiko

- verzögerte Rekonvaleszenz

\section{Physiologische Altersveränderungen}

Neben dem Alter und der Multimorbidität können auch die physiologischen Altersveränderungen Funktionseinbußen verursachen und somit die diagnostischen und therapeutischen Überlegungen verzögern. So nehmen z.B. das Hörund Sehvermögen langsam ab, Muskelmasse reduziert sich und damit die Kraft- und Ausdauerleistungsfähigkeit. Die Festigkeit und Mineralisation der Knochenmasse nehmen im Alter ab und es entsteht eine erhöhte Frakturneigung. Degenerative knöcherne Veränderungen können mit arthrotischen Prozessen einhergehen, die die Mobilität und Selbstständigkeit weiter einschränken. 
Gedächtnisstörungen und somit reduzierte kognitive Fähigkeiten führen u.U. dazu, dass Therapien nicht ausreichend umgesetzt werden können, Medikamente nicht regelmäßig eingenommen oder unzureichend Nahrung und Flüssigkeit aufgenommen werden. Die Folge ist oftmals eine Verschlechterung des funktionellen und medizinischen Zustands. Selbst reduziertes Hunger- und Durstgefühl, die häufig auftreten und hormonell gesteuert sind, können schon ausreichen, um eine mangelhafte Versorgung mit Flüssigkeit und Nährstoffen zu bedingen und so im Rahmen einer Exsikkose notwendige therapeutische und medizinische Anwendungen zu erschweren. Die damit einhergehende Mangelernährung führt zu weiterer körperlicher Reduktion und addiert sich zu den anderen genannten Problemen in der Behandlung älterer Menschen.

Die Veränderungen im Alter können grundsätzlich jedes Organsystem betreffen und in unterschiedlicher Weise Einfluss auf den körperlichen und geistigen Zustand nehmen und so Erkrankungsverläufe verändern und komplizieren.

\section{Geriatrische Syndrome}

Geriatrische Syndrome sind Beschwerdebilder oder auch Krankheitsmuster, die einen Symptomkomplex darstellen, der oftmals als eigenständige Erkrankung oder Diagnose in der klassischen Schulmedizin nicht vorkommt ( $\triangleright$ Tab. 1).

Die Hauptsyndrome, die sogenannten Geriatrischen Giganten, sind Gebrechlichkeit (Frailty-Syndrom), Sarkopenie, Anorexie im Alter und kognitive Dysfunktion [25]. Obwohl diese Hauptsyndrome häufig auftreten, sind sie nicht immer allen an der Behandlung beteiligten Berufsgruppen bekannt.

- Tab. 1 Geriatrische Syndrome

\begin{tabular}{|c|c|}
\hline $\begin{array}{l}\text { Hauptsyndrome } \\
\text { (Geriatrische Giganten) }\end{array}$ & $\begin{array}{l}\text { Weitere geriatrische } \\
\text { Syndrome }\end{array}$ \\
\hline $\begin{array}{l}\text { Gebrechlichkeit (Frailty- } \\
\text { Syndrom) }\end{array}$ & $\begin{array}{l}\text { Mehrfachmedikation } \\
\text { (Polypharmazie) }\end{array}$ \\
\hline $\begin{array}{l}\text { Sarkopenie (Abbau von } \\
\text { Muskelmasse) }\end{array}$ & chronische Schmerzen \\
\hline Anorexie im Alter & Depression, Angststörung \\
\hline \multirow[t]{4}{*}{ kognitive Dysfunktion } & Immobilitätssyndrom \\
\hline & $\begin{array}{l}\text { starke Sehbehinderung und } \\
\text { ausgeprägte } \\
\text { Schwerhörigkeit }\end{array}$ \\
\hline & Inkontinenz \\
\hline & $\begin{array}{l}\text { Sturzsyndrom (Sturznei- } \\
\text { gung und Schwindel) }\end{array}$ \\
\hline
\end{tabular}

\section{Geriatrische Giganten}

Das Frailty-Syndrom bezeichnet eine herabgesetzte körperliche Belastbarkeit bei vermindertem Kraftzustand. Es stellt eine kritische Einschränkung der körperlichen und psychischen Funktionsreserven dar, woraus ein erhöhtes Risiko für weitere oder zunehmende gesundheitliche und funktionelle Verschlechterungen entsteht. Diese können mit ungewollten Krankenhauseinweisungen, dauerhafter oder vermehrter Pflegebedürftigkeit und erhöhter Sterblichkeit einhergehen [7][13][39]. Eine eindeutige Definition hat sich wissenschaftlich noch nicht durchgesetzt. Die Kriterien ähneln sich aber und reichen von diffuser Begrifflichkeit, wie starker Erschöpfung und eingeschränkter Vitalität [13], bis zu reduzierter körperlicher Aktivität und verlangsamter Gehgeschwindigkeit, aber auch zu unfreiwilligem Gewichtsverlust und geringer Muskelkraft [39].

Das Frailty-Syndrom ist nicht mit Multimorbidität gleichzusetzen und ist unabhängig von Anzahl und Art der Vorerkrankungen. Es steht für das gesteigerte Risiko für einen ungünstigen Outcome bei geringer werdenden Reserven physiologischer Systeme [7]. Untersuchungen gehen von einer Häufigkeit der Gebrechlichkeit von bis zu $12 \%$ aller über 65-Jährigen aus [32]. Bei uneinheitlicher Definition ist die Streubreite allerdings hoch (etwa um 3\% nach anderen Definitionen [6]).

Ein weiteres Phänomen, das auch unabhängig von bestehenden Erkrankungen auftritt, ist die Sarkopenie. Hierbei handelt es sich um den vermehrten Verlust von Skelettmuskelmasse und Muskelkraft, der mit Kraft- und Leistungseinbußen einhergeht [9]. Er ist das Ergebnis von Alterungsprozessen verschiedener biologischer Systeme, die sich vor allem an der quergestreiften Muskulatur manifestieren. Ein größeres Risiko für muskuläre Funktionseinschränkung, Sturzgefahr und erhöhte Mortalität ist beschrieben. Mit etwa 20\% der über 70-Jährigen [2] ist die Sarkopenie als häufig zu betrachten und muss in die komplexe Therapiestrategie eingebunden werden ( $\triangleright$ Abb. 1).

Die Anorexie im Alter ist definiert als Appetitlosigkeit und/oder als abnehmende Nahrungsaufnahme im Alter [22]. Die endokrinen Veränderungen, die mit Anorexie, Fehl- und Mangelernährung einhergehen, tragen zumindest zur Sarkopenie bei. Es bestehen also große Schnittmengen zwischen den einzelnen Syndromen. Aufgrund der zentralen Bedeutung der Muskelkraft für die Mobilität und damit Selbstständigkeit ist die Sarkopenie als ein Hauptmerkmal des Frailty-Syndroms zu verstehen. Eine gute Intervention ist nachweislich körperliches Training mit einer optimierten Ernährung [29].

Unter kognitiver Dysfunktion werden im höheren Alter Beeinträchtigungen von Gedächtnis, Aufmerksamkeit und Denkvermögen bezeichnet. Dazu zählen Verwirrtheitszustände bei Delir, mit und ohne Demenzsyndrom 

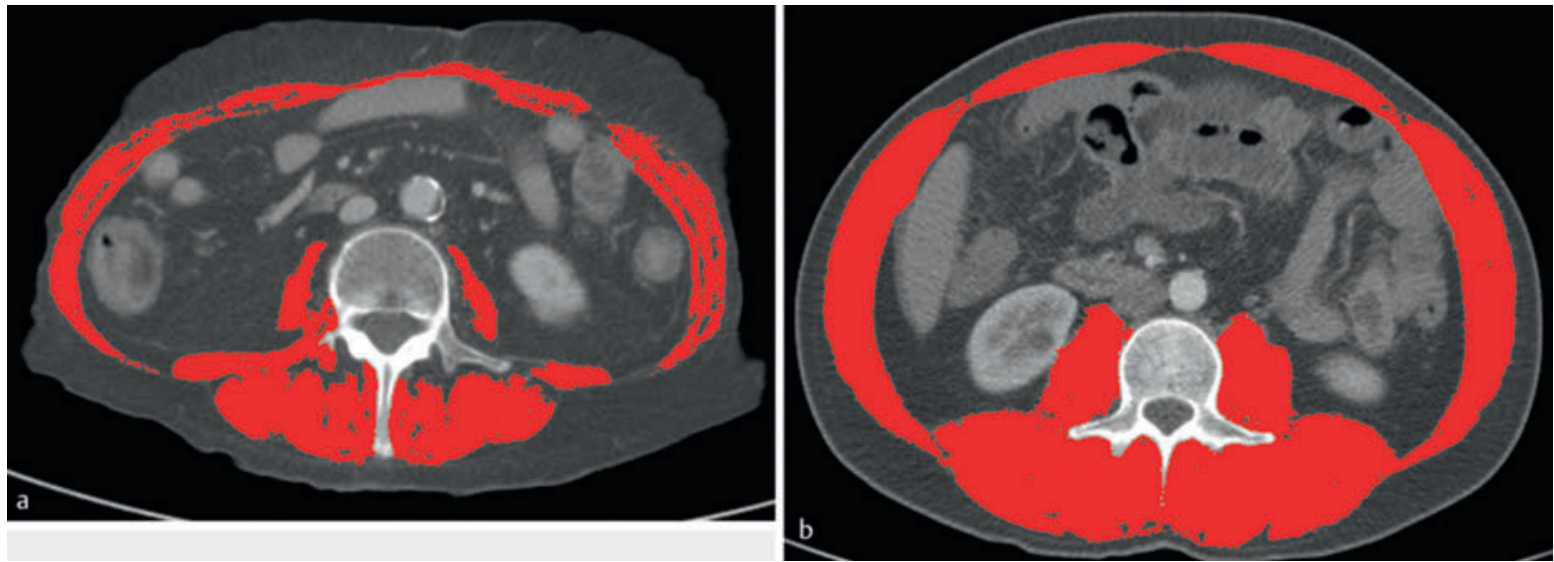

- Abb. 1 Sarkopenie. Transversalschnitte in Höhe der Mitte von LWK3. Rot gefärbt ist die Skelettmuskulatur. a Patient mit Sarkopenie; b Patient ohne Sarkopenie. (Quelle: Plauth M. Ernährung bei multimorbiden Patienten: Daten oder Meinungen? Aktuelle Ernährungsmedizin 2017; 42(5):380-387. doi:10.1055/s-0043-118271)

und unter Medikamentenwirkung, oder Zustände, die z. B. mit einer Hypoxämie einhergehen. Dies kann ebenso eine chronisch-obstruktive Lungenerkrankung (COPD) wie eine Herzinsuffizienz sein. Die Ursachen sind dabei meist multifaktoriell.

\section{Weitere geriatrische Syndrome}

Das Sturzsyndrom ist die häufigste Unfallursache bei über 65-Jährigen. Neben den o.g. Veränderungen, die mit verringerter muskulärer Kraft und eingeschränkter Balancefähigkeit einhergehen, können dabei auch verminderte Hör- und Sehfähigkeit und die durch nervale Veränderungen herabgesetzte Reaktionsfähigkeit eine Rolle spielen [12][28].

Ein ebenfalls häufiges Syndrom ist die Polypharmazie (= Einnahme von fünf oder mehr Arzneimitteln). Sie ist mit erhöhtem Risiko für Wechselwirkungen und Nebenwirkungen verbunden [15][21][42]. Im Alter von 65-79 Jahren nehmen 96,7\% der Frauen und 92,6\% der Männer mindestens ein Arznei- oder Nahrungsergänzungsmittel ein [10]. Die Verordnungstätigkeit ist bei 85 -94Jährigen am höchsten [8]. Dabei kann man von notwendiger Polypharmazie sprechen, wenn tatsächlich mehrere akut zu behandelnde Erkrankungen vorliegen. Durch physiologische Veränderung der Nieren- und Leberfunktion und durch Wechselwirkung der Medikamente können diese verzögert oder verstärkt ausgeschieden bzw. verstoffwechselt werden. Es kann durch Akkumulation eines Wirkstoffs also zu einer verstärkten Wirkung oder durch veränderte Stoffwechselprozesse zu einer abgeschwächten Reaktion kommen.

\section{Geriatrische Assessments}

Geriatrische Assessments dienen der Objektivierung von Fähigkeitsstörungen wie eingeschränkter Selbsthilfefähigkeit, Mobilitätseinschränkung, Sturzgefährdung oder kognitiven Defiziten bzw. depressiven Episoden. Die Untersuchungen zielen aber nicht nur auf das Erkennen vorhandener Defizite ab. Sie sollen auch vorhandene Ressourcen entdecken, um mit diesen die medizinischen und therapeutischen Ansätze zu verbessern.

Um hierbei dieselbe Sprache zu nutzen und möglichst viele Informationen zu erhalten, sollten die Begriffe und Klassifizierungen der ICF (International Classification of Functioning, Disability and Health der WHO) genutzt werden. Hier werden Defizite und mögliche Ressourcen, Funktionsfähigkeit und Förderfaktoren klassifiziert. Begriffe wie Teilhabe und Partizipation sind zentral und beschreiben die Leistungsfähigkeit bzw. die eingeschränkte Funktion, auch in Bezug auf bestimmte Lebenssituationen, in denen sich die Patienten gerade befinden. Auch Umweltfaktoren und personenbezogene Faktoren fließen in die Beurteilung ein. So ist eine berufsübergreifende Kommunikation möglich und erlaubt einen ressourcenorientierten biopsychosozialen Ansatz. Das ist notwendig, um neben der eigentlichen medizinischen Therapie auch die Lebensqualität, Mobilität, Funktionalität u.a. zu beurteilen und die Behandlung zu planen [40].

\section{Damals und heute}

In der Medizingeschichte wurde von M. Warren erstmals eine strukturierte Untersuchung, die körperliche Funktionsfähigkeit, Inkontinenz und geistige Fähigkeiten beinhaltete, beschrieben [38]. Rubenstein et al. [30] zeigten die Verbesserung der Qualität in der ärztlichen Versorgung durch Einführung von Assessmentuntersuchungen, die auf den Untersuchungstechniken von Warren basieren und bis heute immer weiter angepasst werden.

Heutzutage werden Assessments nach unterschiedlichen Zielen in Stufen unterteilt ( $\triangleright$ Abb.2): 


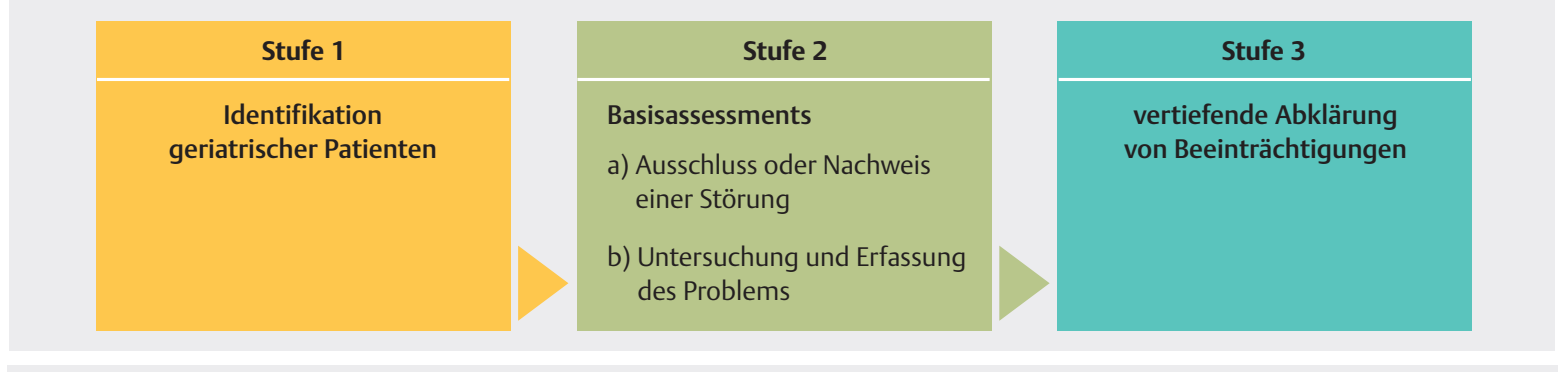

- Abb.2 Assessment-Stufen. (Quelle: N. Pfundtner und F. Blömker)

- Assessments der Stufe 1 dienen der Identifikation geriatrischer Patienten (Screening nach Lachs, ISARidentification of seniors at risk [16]).

- Assessments der Stufe 2 werden in zwei Unterstufen a und b aufgeteilt und Basisassessments genannt. Der Ausschluss/Nachweis einer Störung erfolgt in Stufe 2a. Untersuchungen und die Erfassung eines interventionsbedürftigen Problems geschehen mittels Stufe-2b-Assessments. Hier kommen z. B. der Barthel-Index, der Mini-Mental-Status-Test, die Geriatrische Depressionsskala, die Handkraftmessung, der Uhrentest/ Watson, der Geldzähltest nach Nikolaus und Tinetti und der Timed-up-and-go-Test zur Anwendung.

- Die Stufe-3-Assessments dienen der vertiefenden Abklärung von Beeinträchtigungen. Zu diesen Assessments sind sehr spezielle Untersuchungen zu zählen, die auf denen der Stufe 2 aufbauen und z. B. die genaue Untersuchung einer Handfunktion beinhalten können [20].

\section{Durchführung}

Die Durchführung der unterschiedlichen Assessments wird durch das geriatrische Team übernommen. Hierzu zählen alle an der Behandlung beteiligten Berufsgruppen mit ihrem jeweiligen spezifischen Wissen: Pflegepersonal, Physiotherapie, Ergotherapie, Logopädie, Psychologie, Sozialarbeiter und Ärzte. Manchmal auch weitere spezialisierte Berufsgruppen, wie Zahnärzte, Stomatherapeuten, Musik- und Maltherapeuten u.a.

\section{Therapeutische Zielsetzung}

Wenn alle genannten Aspekte erfasst wurden, muss zunächst ein individuell adaptiertes Ziel mit dem Patienten erarbeitet werden. Zentral sind dabei der
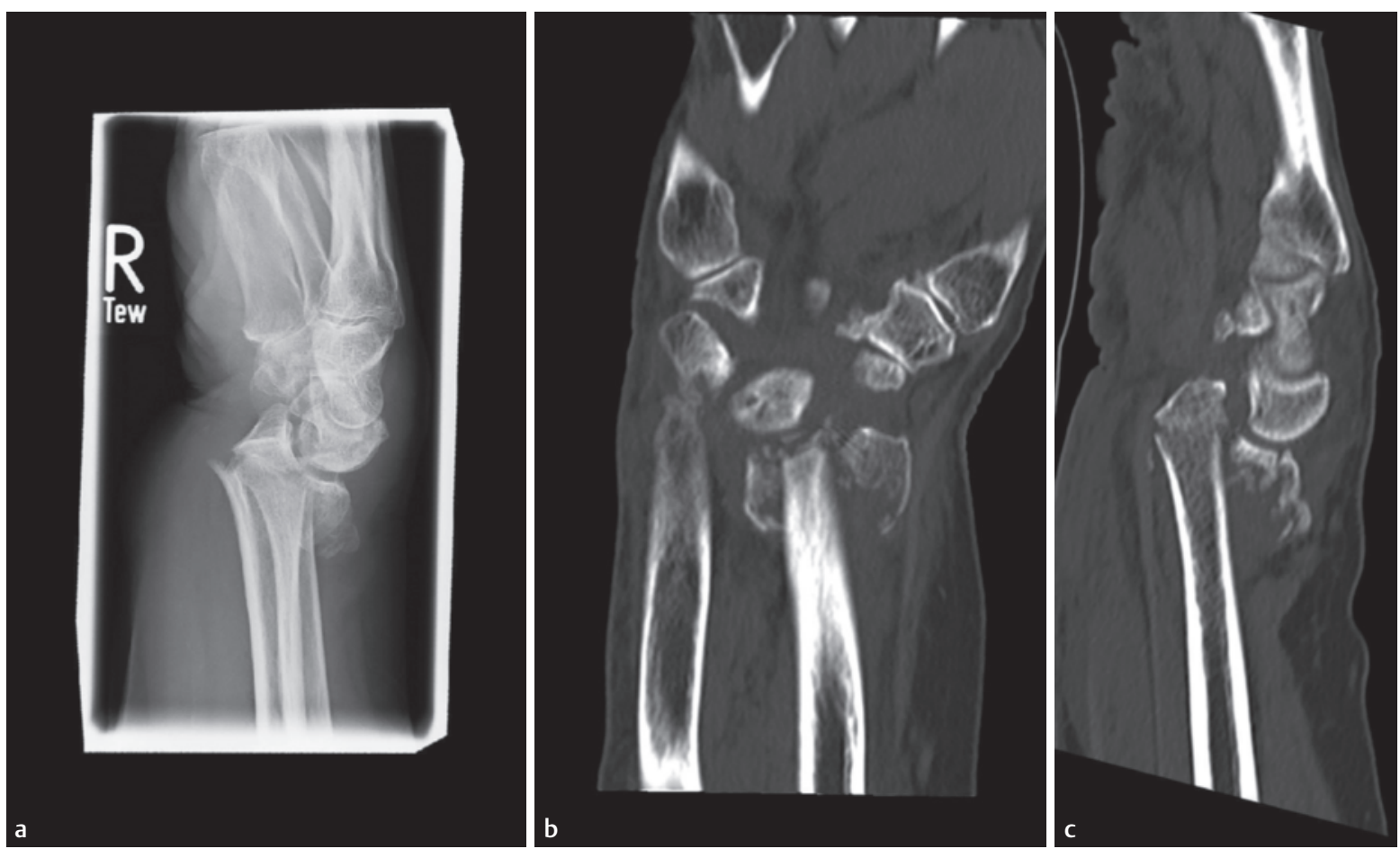

- Abb. 3 Präoperative Bildgebung einer distalen Radius-Extensionsfraktur. (Quelle: F. Blömker); a Präoperatives Röntgenbild, laterale Ansicht; b Repräsentatives CT-Bild, dorsopalmare Ansicht; c Repräsentatives CT-Bild, laterale Ansicht. 


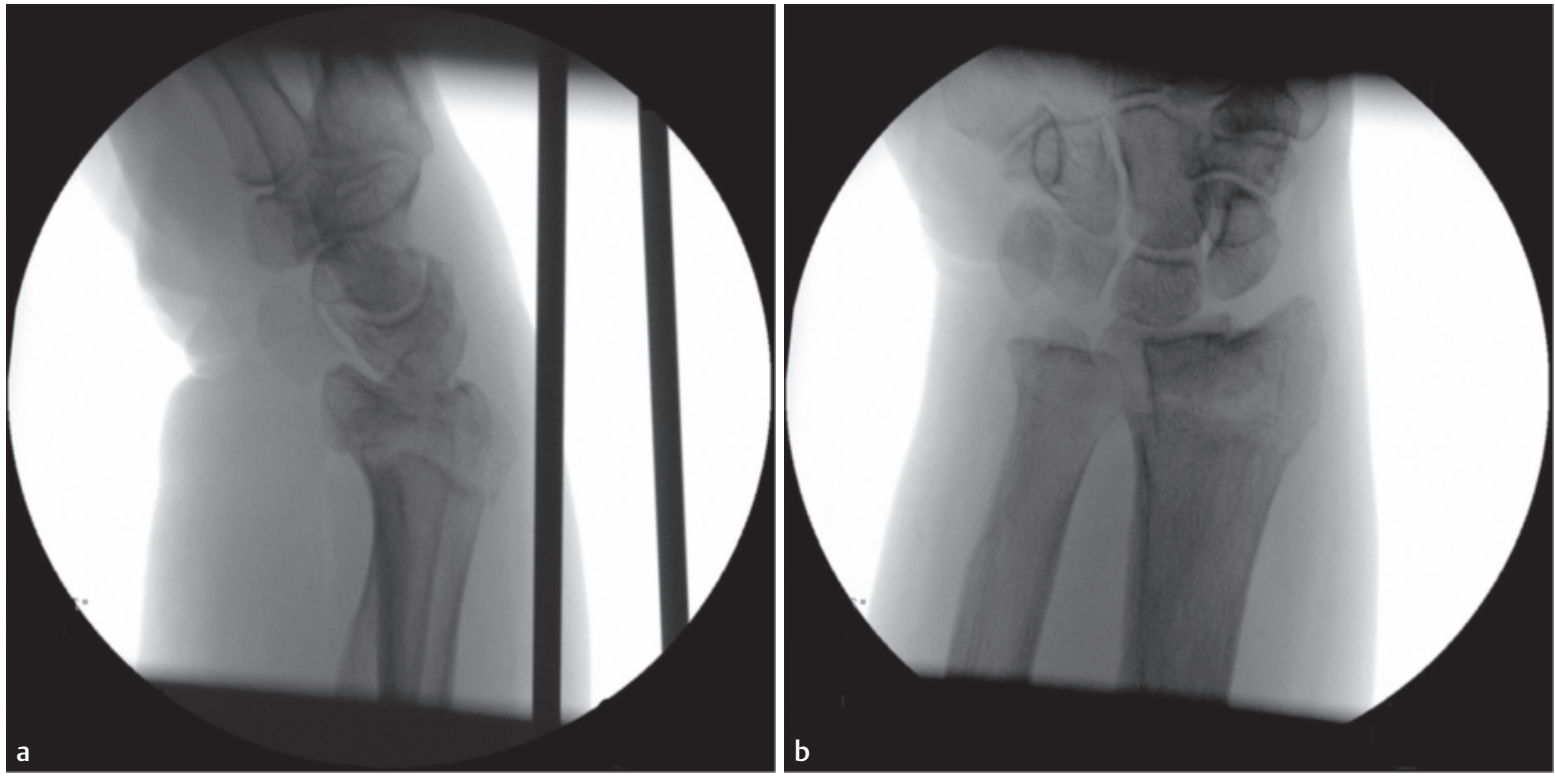

Abb.4 Röntgenbild der im Durchhang reponierten Radiusfraktur. (Quelle: F. Blömker); a Laterale Ansicht; b Dorsopalmare Ansicht.
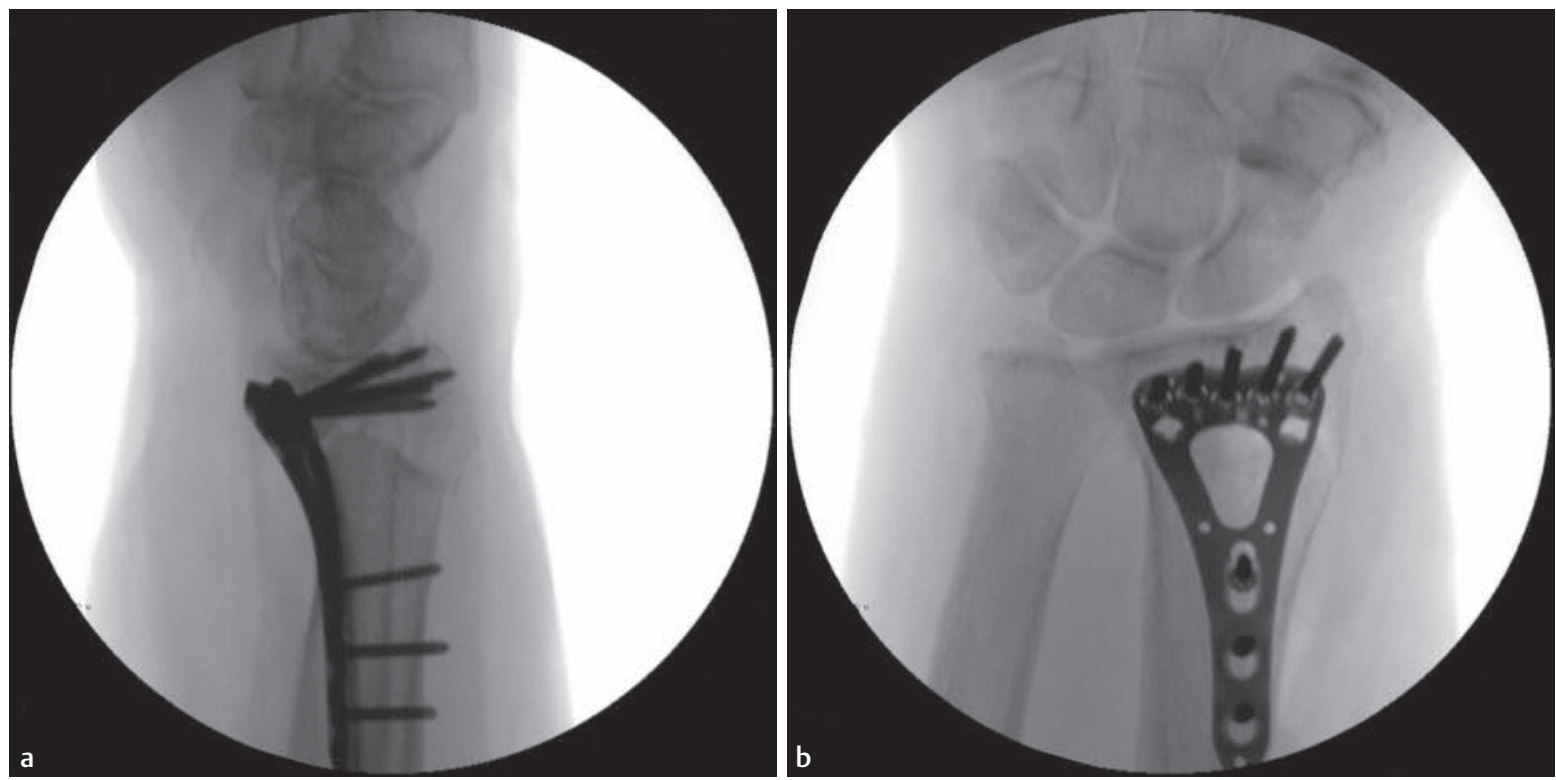

Abb. 5 Intraoperativer Befund nach palmarer Plattenosteosynthese. (Quelle: F. Blömker); a Laterale Ansicht; b Dorsopalmare Ansicht.

Mobilisationserhalt, die Selbsthilfefähigkeit, Kognition und soziale Situation. Wesentlicher weiterer Behandlungsbaustein sind die Motivation zur Eigentherapie und-bedingt durch rezidivierende Sturzereignisse-der Abbau von Sturzangst und nachfolgender Immobilität. Der Mobilitätszuwachs ist deshalb allein eine der wichtigsten Präventionsmaßnahmen.

Optimal ist es, wenn die Kompetenzen unterschiedlicher Fachrichtungen synergistisch genutzt werden können. So kann eine weiterführende Betreuung einzelner
Fachrichtungen als Ergebnis aus einer umfassenden multiprofessionellen Behandlung hervorgehen.

Im klinischen Alltag sind verlässliche, vertrauensvolle Absprachen zwischen den betroffenen verschiedenen medizinischen Fachabteilungen (meist Unfallchirurgie, Geriatrie, Anästhesie) und den verschiedensten Berufsgruppen von herausragender Bedeutung. Eine Analyse der Ausgangmobilität erfolgt hier allerdings erst später durch Fremdanamnesen betreuender Angehöriger, regelhaft aber nicht bei Heimbewohnern. Dabei kann diese Analyse ja nur der 
Ausgangspunkt für die zielgenaue Planung der Rückgewinnung der Mobilität sein. Der Schadenszeitpunkt Sturz mit immobilisierender Fraktur ist nicht selten der Hauptgrund für Heimeinweisungen, Erhöhungen von Pflegegraden oder eskalierender internistischer Komorbidität.

\section{Behandlungsmaßnahmen an Hochbetagten}

Sie unterscheiden sich ganz grundsätzlich von der Behandlung jüngerer Patienten. Abweichungen betreffen den Umfang von Begleiterkrankungen, eine hohe postoperative Komplikationsrate und das kritische Umfeld einer möglichen sozialen Rehabilitation. Gelegentlich spielen auch erhebliche ethische Fragen in Entscheidungsfindungen eine Rolle. Kann z.B. eine Demenz ein Grund dafür sein, notwendige operative Versorgungen nicht durchzuführen?

Therapeutischen Maßnahmen müssen an die Alltagsfunktion und die sozialen Partizipationsmöglichkeiten angepasst werden. Sie müssen in hohem Maße individuell, umfassend und zeitgleich präventiv ausgerichtet sein, um Wirkkraft zu erlangen. Die Einbindung der an der Behandlung beteiligten Mitarbeiter in therapeutischen Teams ist nicht nur im innerklinischen Bereich, sondern auch in Praxen wünschenswert. Sie ist ein wesentlicher Bestandteil der Therapie. Empfehlenswert ist eine enge Rückkopplung zwischen Therapeuten und Ärzten. Frühe rehabilitative Ansätze der Nachbehandlung werden häufig erst spät in den Therapieverlauf eingebunden und sind dann eher schwerer umsetzbar. Eine enge Einbindung betreuender Angehöriger-insbesondere bei kognitiven Defiziten-ist bereits zu Beginn der Behandlungsmaßnahmen sinnvoll.

\section{Distale Radiusfraktur beim alten Menschen}

Die Abnahme von Knochendichte, insbesondere des abstützenden kortikalen Knochens sowie des Muskelmantels (Sarkopenie), ist meist ein physiologischer Prozess des Alterns. Wiederherstellende Maßnahmen müssen also in erheblichem Umfang diesen Voraussetzungen genügen. Bedeutend sind insbesondere funktionelle Aspekte einer möglichen frühzeitigen Krafteinleitung auf den Karpus bei axialer Stauchung der Radiuskonsole-wie dies beispielsweise beim Nutzen eines Rollators, bei funktionellen Geheinschränkungen oder beim Umgreifen von Gehhilfen erforderlich wird. Die Kraftreserven sind bei Hochbetagten sehr unterschiedlich. Der Rückgewinn der Ausgangsmobilität für die Mobilisation ohne/mit Hilfen (Rollator/Rollstuhl/Pflegestuhl) ist dabei kein Selbstzweck. Für die Versorgung sind die umfassenden Anforderungen möglichst ohne weitere Risiken für die Patienten jeweils individuell abzustimmen.

Operative Versorgung des Handgelenks

Am Handgelenk empfiehlt sich, aufgrund der besonderen Bedeutung der Abstützkraft im Alter, bei Vorliegen einer Fraktur oftmals die operative Versorgung - abhängig vom Dislokationsausmaß. Auch bei mittel- und langfristig guten Ergebnissen der konservativen Behandlung haben sich operative Verfahren in der Gruppe alterstraumatologischer Patienten bewährt. Denn was sind langfristige Ergebnisse bei dieser Patientengruppe?

Durch den Einsatz winkelstabil verriegelbarer Formplattenosteosynthesen kann vorzugsweise auf der palmaren Seite eine Kraftbelastung meist frühzeitig erlaubt werden. Zwar führen auch geschlossene Repetitionen und K- Draht-Fixationen häufig zu zufriedenstellenden radiologischen Stellungskontrollen. Diese erlauben jedoch keinesfalls frühfunktionelle Belastungen.

Die Bilderstrecke zeigt das Handgelenk eines 85 Jahre alten Patienten, der beim nächtlichen Toilettengang im häuslichen Milieu stürzte. Die Bildgebung (Röntgen und $\mathrm{CT}$ ) zeigte eine distale Radius-Extensionsfraktur ( Zunächst reponierte der Chirurg diese im Durchhang ( Abb.4), konnte jedoch keine zufriedenstellende, stabile Stellung erreichen. So versorgte er die Radiusfraktur mit winkelstabiler, palmarer Platte ( $\mathbf{A} \mathbf{A b} \mathbf{b} . \mathbf{5})$. Es folgten eine gipsfreie Nachbehandlung und sofortige Mobilisation am Rollator (Nebendiagnose Z.n. Femurfraktur). Er trug vier Wochen eine Handgelenkorthese.

\section{Konservative Behandlung des Handgelenks}

Für die konservative Behandlung eignen sich vorzugsweise kognitiv geordnete Patienten mit stabil zu retinierenden Frakturen und Gangstabilität. Selbst additiv mit palmaren Castverstärkungen ist eine zuverlässige Druckentlastung meist nicht gewährleistet. Insofern ist eine enge klinische und radiologische Überwachung dieser Patienten erforderlich. In der Praxis ist jedoch durch die stark regional bestehende Überalterung ein geordneter Zugang zu Nachuntersuchungen erschwert, ebenso finden nach knöcherner Konsolidierung nur unzureichende oder sehr zeitverzögerte Re-Mobilisierungen statt. Häufig erst bei Vorliegen von Komorbidität.

\section{Behandlungsalgorithmus für operativ versorgte Radiusfrakturen}

In der Nachbehandlung ist eine möglichst strukturierte, frühe Therapie anzustreben. Klinisch hat sich folgender Behandlungsalgorithmus für Nachbehandlung operativ versorgter Radiusfrakturen bewährt:

- zweitägliche Wundkontrollen, milde Lymphdrainage, Spiegeltherapie

- Fadenzug am zehnten postoperativen Tag

- aktive Bewegungen aller Gelenke von Langfinger und Daumen, Griffqualitäten sollen erreicht werden, Flexions-Extensionsübungen, Pronation und Supination passiv

- ab der vierten Woche moderate Gewichtsbelastung

- Ziel: volle Gewichtsbelastung ab der sechsten Woche 


\section{Funktion vor Form}

Das Prinzip Funktion vor Form in der Behandlung alterstraumatologischer Patienten lässt große Spielräume in der Nachbehandlung zu. Vor dem Hintergrund eher allgemein gehaltener, rigider Behandlungsempfehlungen muss jeweils hinterfragt werden, welche Therapieziele erreicht werden sollen und was der Patient dafür von uns benötigt. Zu berücksichtigen ist, dass der Zugang zu Therapeuten abhängig ist von der Wohnortnähe, bei regionsspezifischen Überalterungen eine zusätzliche Verschärfung des Problems. Mobile geriatrische Rehabilitationsmöglichkeiten sind in der Zukunft demnach vonnöten. Der systematische Aufbau von individuell abgestimmten Behandlungsbausteinen schafft für Patienten, Zuweiser, Angehörige und Kostenträger gute Rahmenbedingungen.

\section{Zusammenfassung}

Komplexe geriatrische Erkrankungsbilder, die mit weiteren chronischen Krankheiten sowie altersphysiologischen Beeinträchtigungen das klinische Bild dominieren, führen dazu, dass bei der Behandlung einer neu aufgetretenen Akuterkrankung bzw. - verletzung eine besondere diagnostische und therapeutische Vorgehensweise notwendig wird. Eine monodimensionale oder auch nur kausale Therapie wird der Situation beim erkrankten alten Patienten nicht gerecht. Neben der Diagnostik und Therapie der Akuterkrankung ist der Erhalt oder die Wiederherstellung der Selbstständigkeit sowie der funktionellen Kompetenz und damit der Autonomie und der Teilhabe vorrangig. Die weiterführende Therapie muss in ein multiprofessionelles Behandlungskonzept eingebunden sein, um die genannten altersspezifischen Risiken und Kontextfaktoren in der Behandlungsstrategie ausreichend zu berücksichtigen.

Da das numerische Alter als prognostischer Faktor nur unzureichend aussagefähig ist, sollte also eine rehabilitative Behandlung den alten Patienten grundsätzlich nicht vorenthalten werden. Das gilt besonders für die Patienten, die zunächst eine Akuterkrankung überstehen müssen. In der Folge profitieren die alten Menschen häufiger von einer den akutstationären Klinikaufenthalt begleitenden Frührehabilitation bzw. von einer sich anschließenden klassischen Rehabilitationsbehandlung.

\section{Autorinnen / Autoren}

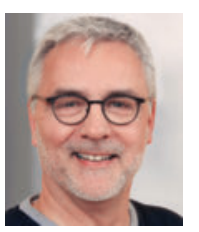

\section{Norbert Pfundtner}

Facharzt für Innere Medizin mit Zusatzbezeichnung Geriatrie. Seit 2012 ist er Chefarzt im St. Rochus Krankenhaus Steinheim/Westf., Klinikum Weser-Egge, KHWE und seit 2018 ärztlicher Direktor im Klinikum Weser-Egge. Er baute eine Klinik für Akutgeriatrie auf, einschließlich alterschirurgischer Station.

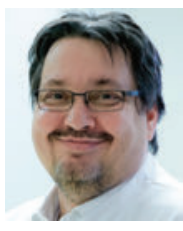

\section{Frank Blömker}

Facharzt für Orthopädie und Unfallchirurgie und seit 2011 im medizinischen Leitungsteam der Akademie für Handrehabilitation. Er ist Chefarzt der Klinik für Unfallchirurgie an der Katholischen Hospitalvereinigung Weser-Egge. Davor war er lange Chefarzt der Abteilung für Unfall - und Handchirurgie und Sporttraumatologie am AGAPLESION Ev. Bathildiskrankenhaus in Bad Pyrmont.

Korrespondenzadresse

St. Rochus Krankenhaus

Nieheimer Str. 34

32839 Steinheim

E-Mail: n.pfundtner@khwe.de

\section{Literatur}

[1] Aman U, Schmedt N, Garbe E. Ärztliche Verordnungen von potenziell inadäquater Medikation bei Älteren: Eine Analyse basierend auf der PRISCUS-Liste. Dtsch Arztebl Int 2012; 109 (5):69-75

[2] Berger M], Doherty T]. Sarcopenia: Prevalence, mechanisms, and functional consequences. In: Mobbs C, Hof P, eds. Body Composition and Aging. Basel: Karger; 2010: 94-114

[3] Böcker W, Kammerlander C, Gosch M, Stumpf UC. Alterstraumatologie: Praxisnah-kompakt-interdisziplinär. Stuttgart: Schattauer / Thieme; 2019

[4] Borchelt M. Besondere Aspekte der Pharmakotherapie im höheren Lebensalter; 2004. Im Internet: www.geriatrie-drg.de/Pub lic/Docs/Pharmakotherapie_Geriatrie_2004_Borchelt.pdf; Stand: 31.10.2019

[5] Budnitz DS, Lovegrove MC, Shehab N et al. Emergency hospitalizations for adverse drug events in older Americans. N Engl J Med 2011; 365(21):2002-2012

[6] Buttery A, Busch M, Gärtner B et al. Prevalence and correlates of frailty among older adults: Findings from the German Health Interview and Examination Survey. BMC Geriatr 2015; 15: 22

[7] Clegg A, Young J, Iliffe S et al. Frailty in elderly people. Lancet 2013; 381: 752-762

[8] Coca V, Nink K. Arzneimittelverordnungen nach Alter und Geschlecht. In: Schwabe U, Hrsg. Arznei-Verordnungsreport 2010. Springer: Berlin; 2010: 933-946

[9] Cruz-Jentoft AJ, Baeyens JP, Bauer JM et al. Sarcopenia: European consensus on definition and diagnosis: Report of the European Working Group on Sarcopenia in Older People. Age Ageing 2010; 39(4):412-423

[10] DEGS1-Studie zur Arzneimittelanwendung. Bundesgesundheitsblatt-Gesundheitsforschung-Gesundheitsschutz 2013. Im Internet: https://www.degs-studie.de/deutsch/ergeb nisse/degs1/degs1-basispublikation.html; Stand: 31.10.2019

[11] Deuschl G, Maier W et al. S3-Leitlinie Demenzen. In: Deutsche Gesellschaft für Neurologie, Hrsg. Leitlinien für Diagnostik und Therapie in der Neurologie. Langversion 2016. Im Internet: www.dgn.org; Stand: 01.11.2019

[12] Deutscher Verkehrssicherheitsrat e. V. Verkehrssicherheitsprogramme in Deutschland. Mobil bleiben, aber sicher! www.ver kehrssicherheitsprogramme.de/site/detail; Stand: 11.02.2015 
[13] Fried L, Tangen C, Walston J et al. Frailty in older adults: Evidence for a phenotype. J Gerontol A Biol Sci Med Sci 2001; 56 (3): M146-M156

[14] Fuchs J, Busch M, Lange $C$ et al. Prevalence and patterns of morbidity among adults in Germany: Results of the German Telephone Health Interview Survey "German Health Update (GEDA) 2009«. Bundesgesundheitsbl-GesundheitsforschGesundheitsschutz 2012; 55: 576-586

[15] Haider $S$, Johnell $\mathrm{K}$, Thorslund $\mathrm{M}$ et al. Trends in polypharmacy and potential drug-drug interactions across educational groups in elderly patients in Sweden for the period 1992 2002. Int J Pharmacol Ther 2007; 45: 643-653

[16] Hofmann W, Nikolaus T, Pientka L, Stuck AE. Arbeitsgruppe Geriatrisches Assessment (AGAST): Empfehlungen für den Einsatz von Assessment-Verfahren. Zeitschrift für Gerontologie und Geriatrie 1995; 28: 29-34

[17] Hohmann E, Meta M, Navalgund V, Tetsworth K. The relationship between radiological alignment of united distal radius fractures and functional and patient-perceived outcomes in elderly patients. J Orthop Surg 2017; 25: 2309499016684976

[18] Karantana A, Downing ND, Forward DP et al. Surgical treatment of distal radial fractures with a solar locking plate versus conventional percutaneous methods: A randomized controlled trial. J Bone Joint Surf Am 2013; 95: 1737-1744

[19] Knopf H, Grams D. Arzneimittelanwendung von Erwachsenen in Deutschland: Ergebnisse der Studie zur Gesundheit Erwachsener in Deutschland (DEGS1). Bundesgesundheitsbl-Gesundheitsforsch-Gesundheitsschutz 2013; 56(5/6): 868-877

[20] Krupp S, Frohnhofen H. S1 LL „Geriatrisches Assessment der Stufe 2“, AWMF RgNr. 084-002. Im Internet: www.awmf.org/ leitlinien/detail/II/084-002.html; Stand: 31.10.2019

[21] Lai S, Liao K, Liao C et al. Polypharmacy correlates with increased risk for hip fracture in the elderly: A population-based study. Medicine 2010; 89: 295-299

[22] Land F, Calvani R, Tosato M. Anorexia of aging: Risk factors, consequences, and potential treatments. Nutrients 2016; 8 (2): 69

[23] Lawton MP, Brody EM. Assessment of older people: Self-maintaining and instrumental activities of daily living. Gerontologist 1969; 9: 179-186

[24] Menning S, Hoffmann E. Report Altersdaten. Die Babyboomer: Ein demografisches Porträt. Berlin: Deutsches Zentrum für Altersfragen; 2009

[25] Morley JE. Geriatricians: The super specialists. J Am Geriatr Soc 2017; 65: 866-868

[26] Plauth M. Ernährung bei multimorbiden Patienten: Daten oder Meinungen? Aktuelle Ernährungsmedizin 2017; 42 (5):380-387. doi:10.1055/s-0043-118271

[27] Rattay P, Butschalowsky H, Rommel A et al. Inanspruchnahme der ambulanten und stationären medizinischen Versorgung in Deutschland. Bundesgesundheitsbl-GesundheitsforschGesundheitsschutz 2013; 56(5/6): 832-844

[28] Robert Koch-Institut, Hrsg. Das Unfallgeschehen bei Erwachsenen in Deutschland. Ergebnisse des Unfallmoduls der Befragung "Gesundheit in Deutschland aktuell« GEDA 2010. Beiträge zur Gesundheitsberichterstattung des Bundes. Berlin: RKI; 2013

[29] Robinson S, Cooper C, Aihie Sayer A. Nutrition and sarcopenia: A review of the evidence and implications for preventive strategies. J Aging Res 2012. doi:10.1155/2012/510801

[30] Rubenstein L, Josephson KR, Wieland GD et al. Effectiveness of a geriatric evaluation unit: A randomized clinical trial. N Engl J Med 1984; 311: 1664-1670

[31] Runge M, Rehfeld G. Geriatrische Rehabilitation im therapeutischen Team. 2. Aufl. Stuttgart: Thieme; 2001

[32] Santos-Eggimann B, Cuenoud P, Spagnoli ] et al. Prevalence of frailty in middle-aged and older community-dwelling Europeans living in 10 countries. J Gerontol A Biol Sci Med Sci 2009; 64(6):675-681

[33] Scherer M. Multimorbidität. DEGAM-Leitlinie, AWMF 053047. Im Internet: https://www.awmf.org/leitlinien/detail/II/0 53-047.html; Stand: 31.10.2019

[34] Statistisches Bundesamt. Bevölkerung Deutschlands bis 2060. 12. koordinierte Bevölkerungsvorausberechnung. Wiesbaden: Destatis; 2009

[35] Statistisches Bundesamt. Statistik der natürlichen Bevölkerungsbewegung: Durchschnittliche Lebenserwartung im Alter von ... Jahren; 2012. Im Internet: www.gbe-bund.de; Stand: 31. 10.2019

[36] Statistisches Bundesamt. Fortschreibung des Bevölkerungsstandes, Bevölkerung zum Stichtag 31.12. des jeweiligen Jahres; 2014. Im Internet: www.gbe-bund.de; Stand: 31.10.2019

[37] Statistisches Bundesamt. Krankenhausstatistik: Diagnosedaten der Patienten und Patientinnen in Krankenhäusern (Sonderauswertung); 2015. Im Internet: www.gbe-bund.de; Stand: 31. 10.2019

[38] Warren MW. Care of the chronic sick: A case for treating chronic sick in blocks in a general hospital. Br Med J 1943

[39] Woods A, Cohen RA, Pahor M. Cognitive frailty: Frontiers and challenges. J Nutr Health Aging 2013; 17(9):741-743

[40] World Health Organization. International classification of functioning, disability and health: ICF. Short Version. World Health Organization 2001. Im Internet: https://apps.who.int/ iris/handle/10665/42417; Stand: 31.10.2019

[41] Zeyfang A, Hagg-Grün U, Nikolaus T. Basiswissen Medizin des Alterns und des alten Menschen. 2. Aufl. Berlin: Springer; 2013

[42] Ziere G, Dieleman J, Hofman A et al. Polypharmacy and falls in the middle age and elderly population. Br J Clin Pharmacol 2006; 61: 218-223 